\title{
Preparation of Stearic acid/Diatomite Composite Phase Change Material
}

\author{
Jianping Zong ${ }^{1}$, Defu Wang ${ }^{1}$, Yanlin Jin ${ }^{1}$, Xing Gao ${ }^{1 *}$, Xinxin Wang ${ }^{1}$ \\ ${ }^{1}$ School of Chemistry and Bioengineering, Qilu Institute of Technology, Jinan, Shandong, 250200, China
}

\begin{abstract}
The composite phase change material was prepared via the impregnation method using diatomaceous as the carrier and stearic acid as the phase change material. The effects of diatomite content, temperature, immersion time and pressure on the mass ratio of stearic acid and diatomaceous earth in the composite phase change materials were discussed. The experimental results showed that the optimum conditions for preparing stearic acid/diatomite composite phase change material were immersion temperature of $80^{\circ} \mathrm{C}$, socking time of $2 \mathrm{~h}$, diatomite mass fraction of $23.04 \%$, and vacuum degree of $0.03 \mathrm{MPa}$. Finally, the infrared spectroscopy analysis of stearic acid/diatomite composite phase change energy storage material showed that there is no chemical reaction between stearic acid and diatomite. And they are held together by intermolecular forces.
\end{abstract}

\section{Introduction}

With the rapid development of industry, the supply of fossil energy is insufficient. In recent years, more and more people have begun to study phase change materials in order to alleviate the energy crisis, since phase change materials can exchange energy with its surroundings during phase change[1]. The main advantage of phase change materials is to improve energy efficiency, followed by relatively stable phase change temperature, which can be widely used in various fields, such as agriculture, textile industry and electronics industry[2].

Phase change materials can be divided into single phase change materials and composite phase change materials according to their composition. The single phase change materials include inorganic phase change materials and organic phase change material. Inorganic phase change materials are extremely corrosive and inconvenient to use although they have high thermal conductivity and good heat transfer effect. Organic phase change materials have high latent heat of phase change and stable performance. However, they have low thermal conductivity and poor heat transfer effect.

Research on composite phase change materials has become a hot spot in order to overcome the defects of the single phase change materials[3]. The composite phase change materials are composed of phase change materials and carrier materials, which have high thermal conductivity and stability[4].

Diatomite is a natural mineral raw material. It has the advantages of large specific surface area, high chemical stability and lower price than activated carbon[5]. And diatomite is widely used as a carrier for composite materials. $\mathrm{Fu}$ et al. prepared shaped phase change composite materials by dipping method with stearic acid and diatomaceous[6]. Sun et al. studied the preparation process and properties of paraffin/modified diatomite composite phase change energy storage materials[7].

In our study, we prepared stearic acid/diatomite composites via the impregnation method. We also studied the effects of diatomite content, temperature, immersion time and pressure on the mass ratio of stearic acid and diatomaceous earth in the composites and the combination of stearic acid and diatomite.

\section{Experiment}

\subsection{Pretreatment of Raw material}

The diatomite was put into the muffle furnace and calcined at $450^{\circ} \mathrm{C}$ for 2 hours. Then the diatomaceous earth was acidified with $70 \%$ sulphuric acid for 4 hours when it cooled to room temperature. The acidified diatomaceous earth was washed several times with distilled water until $\mathrm{pH} \approx 7$. The solution was subjected to suction filtration on a vacuum pump. The damp diatomaceous earth was put into an evaporating dish and dried in a drying oven for 24 hours.

\subsection{Preparation of Diatomite Composite Phase Change Material}

We put an appropriate amount of stearic acid and ethanol into a flask. The flask was heated and stirred until the stearic acid was completely melted. Then we added an appropriate amount of diatomite into the flask. The solvent was separated after stirring at a certain temperature for a

*e-mail: gaoxing620@qlit.edu.cn 
certain time. The composite phase change materials can be obtained by drying for 24 hours at $50^{\circ} \mathrm{C}$ in a vacuum drying oven. We evaluated the influence of diatomite content, the temperature, soaking time and the degree of vacuum based on the mass ratio of stearic acid to diatomite in composite.

\subsection{Infrared spectrum analysis}

Infrared spectrometer (Nicoletis5, Thermo Scientific) was used to determine the infrared spectrum of diatomite and stearic acid/diatomite composite.

\section{Results and discussion}

\subsection{Effect of diatomite content on stearic acid content in composite}

We prepared the composite material with different diatomite content which immersed in a water bath at $80^{\circ} \mathrm{C}$ for 2 hours under normal pressure. We plotted the influence of diatomite content on stearic acid content in the composites.

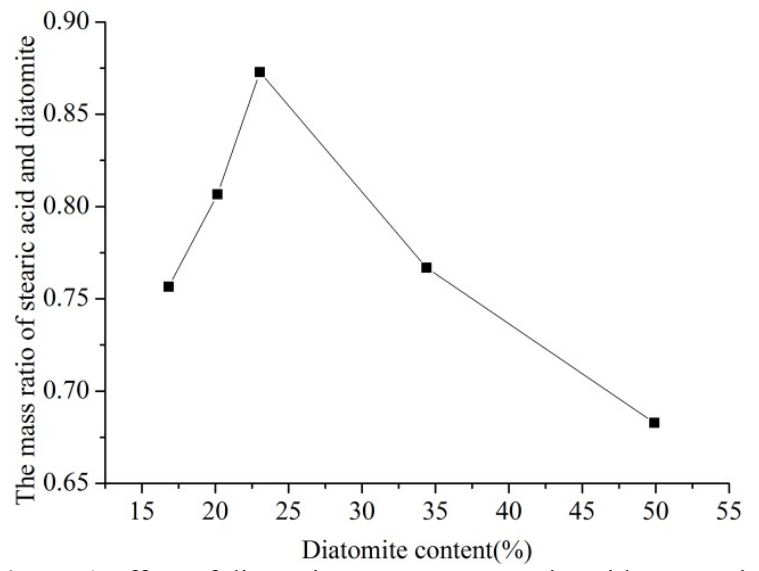

Figure 1 Effect of diatomite content on stearic acid content in composites

The mass ratio of stearic acid to diatomite increased first and then decreased with the change of diatomite content, as the line demonstrated in Figure 1. The reason may be that the more the amount of diatomaceous earth, the larger the volume of the microporous that can hold stearic acid, resulting in the more stearic acid was adsorbed when the amount of stearic acid was stable. However, the amount of adsorbed stearic acid did not increase with the increase of diatomite when all of stearic acid was adsorbed. As a result, the mass ratio of stearic acid to diatomite decreased with the amount of diatomite increasing. It can be seen that the optimal diatomite content for stearic acid/diatomite composite was $23.04 \%$.

\subsection{Effect of temperature on stearic acid content in composite}

We prepared the composite material with diatomite content of $23.4 \%$ which immersed in a water bath at different temperature for 2 hours under normal pressure. We plotted the influence of temperature on stearic acid content in the composite materials.

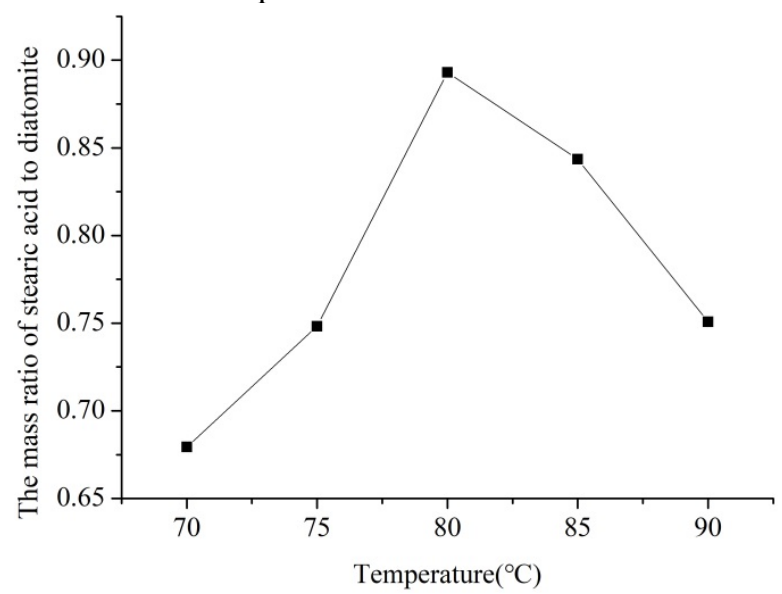

Figure 2 Effect of temperature on stearic acid content in composites

It can be concluded from the Figure 2 that the mass ratio of stearic acid to diatomite increased first and then decreased with temperature rising. This may be because the viscosity of stearic acid decreased as the temperature rising. And it was easier for stearic acid molecules to enter the internal pores of diatomite. However, the volatilization of stearic acid gradually increased which would reduce the amount of stearic acid adsorbed when the temperature was too high. It can be seen that the optimal temperature for preparing stearic acid/diatomite composite was $25^{\circ} \mathrm{C}$.

\subsection{Effect of time on stearic acid content in composite}

We prepared the composite material with diatomite content of $23.4 \%$ which immersed in a water bath at $80^{\circ} \mathrm{C}$ for different time under normal pressure. We plotted the influence of time on stearic acid content as shown in Figure 3.

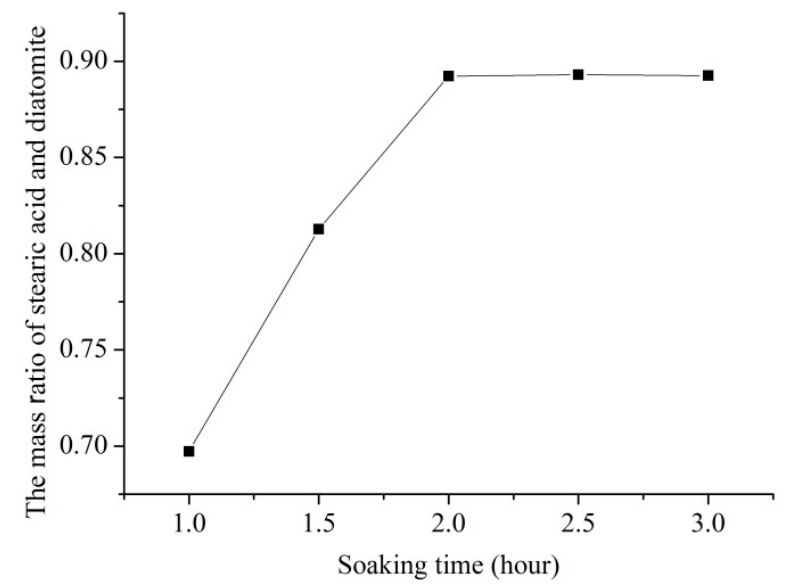

Figure 3 Effect of soaking time on stearic acid content in composites

It can be concluded from the Figure 3 that the mass ratio of stearic acid and diatomaceous earth increased first and then remained unchanged with extension of the immerse time. The reason may be that the microporous in the diatomite were stable when the mass of diatomite was constant. More and more stearic acid was filled into the microporous of diatomite with time. The amount of stearic acid adsorbed was unchanged when all of the microporous 
were filled while the time is continuously extended. And the mass ratio of stearic acid to diatomite remained unchanged two hours later. The optimum socking time was two hours with comprehensive consideration of various factors.

\subsection{Effect of pressure on stearic acid content in composite}

We prepared the composite material with diatomite content of $23.4 \%$ which immersed in a water bath at $80^{\circ} \mathrm{C}$ for 2 hours under different pressure. We plotted the influence of pressure on stearic acid content as shown in Figure 4.

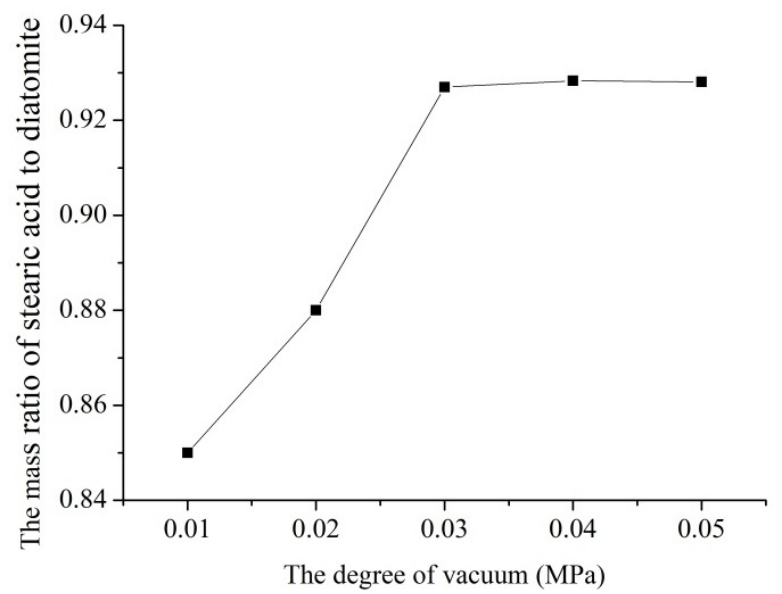

Figure 4 Effect of pressure on stearic acid content in composites

We can come to the conclusion that the mass ratio of stearic acid to diatomaceous earth increased first and then remained unchanged as the vacuum degree of the reaction device increases. This may be because the higher the vacuum, the less air in the microporous of diatomite, which maked it easier to absorb more stearic acid molecules. However, the mass ratio of stearic acid and diatomaceous earth remained unchanged despite the increase in vacuum when the stearic acid filled all of the microporous of diatomaceous earth. The optimum degree of vacuum pressure was $0.3 \mathrm{MPa}$ with comprehensive consideration of various factors.

\subsection{Infrared Spectral Characterization}

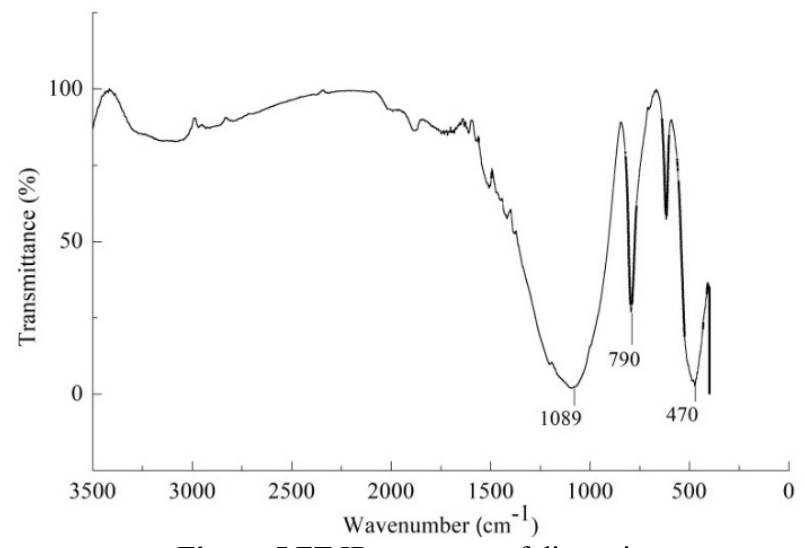

Figure 5 FT-IR spectrum of diatomite

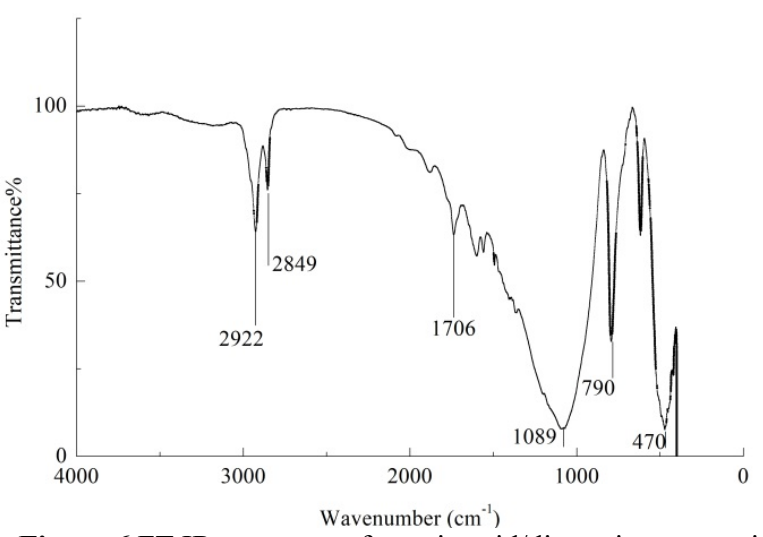

Figure 6 FT-IR spectrum of stearic acid/diatomite composite material

Infrared spectra of diatomite and the composite phase change material were shown in Figure 5 and Figure 6. The absorption peaks of typical diatomite at $1089 \mathrm{~cm}^{-1}[\mathrm{Si}-\mathrm{O}$ asymmetrical stretching], $790 \mathrm{~cm}^{-1}$ [Si-O-Si symmetrical stretching] and $470 \mathrm{~cm}^{-1}$ [Si-O bending] can be seen clearly in the two spectra. Furthermore, the peaks of stearic acid of the composite at $2922 \mathrm{~cm}^{-1}$ and $2849 \mathrm{~cm}^{-1}[\mathrm{v}(\mathrm{C}-\mathrm{H})]$, $1705 \mathrm{~cm}^{-1}[\mathrm{v}(\mathrm{C}=\mathrm{O})]$, and $930 \mathrm{~cm}^{-1}(\mathrm{O}-\mathrm{H}$ rocking $)$ indicated that stearic acid was adsorbed into the diatomite in Figure 6. Compared Figure 5 with Figure 6, we can come to the conclusion that no new chemical bond was formed. Therefore, it can be known that the stearic acid and diatomite in the composite material were bound together by intermolecular force.

\section{Conclusion}

The stearic acid/diatomite composite phase change material was successfully prepared by the impregnation method. The optimum conditions for preparing stearic acid/diatomite composite phase change material were immersion temperature of $80^{\circ} \mathrm{C}$, stirring time of $2 \mathrm{~h}$, diatomite mass fraction of $23.04 \%$, and vacuum degree of $0.03 \mathrm{MPa}$. The infrared spectroscopy analysis of stearic acid/diatomite composite showed that there was no chemical reaction between stearic acid and diatomite. They were held together by intermolecular forces.

\section{Acknowledgments}

This work was supported by Shandong Province University Student Innovation Training Project (project number S202013998009).

\section{References}

1. Safari A., Saidur R., Sulaiman F.A., et al. (2017) A review on super cooling of phase change materials in thermal energy storage systems. Renewable and Sustainable Energy Reviews, 70: 905-906.

2. Wang W.W., Song X.F., Cai Y.B., et al. (2019) Preparation of diatomite-based shape-stabilized PCM and its thermal property. New Chemical Materials, 47: 228-231. 
3. Zhao S.X., Yan H., Li Y.T., et al. (2017) Research on properties of expanded graphite-based stereotyped phase change materials. Contemporary Chemical Industry, 46: 2038-2041.

4. Jin Y., Lee W., Musina Z., et al. (2010) A one-step method for producing microencapsulated phase change materials. Particuology, 8: 588-590.

5. Wang j., Zheng S.L. (2012) Research on preparation technology of Diatomite composite phase change material. Non-metallic Mines, 35: 55-57.

6. Fu X., Liu Z., Wu B., et al. (2016) Preparation and thermal properties of stearic acid/diatomite composites as form-stable phase change materials for thermal energy storage via direct impregnation method. Journal of Thermal Analysis \& Calorimetry, 123:1173-1181.

7. Sun Y.Z., Xi G.X., Yang W.J. (2010) Preparation and performance of paraffin/modified diatomite composite phase change energy storage material. New Chemical Materials, 38: 46-48. 